\title{
Is there an association between labor induction and attention-deficit/hyperactivity disorder among children?
}

\author{
Ensiyeh Jenabi, $\mathrm{PhD}^{1}$, Mahdieh Seyedi, $\mathrm{MSc}^{1}$, Saeid Bashirian, $\mathrm{PhD}^{2}$, Bita Fereidooni, $\mathrm{PhD}^{3}$ \\ ${ }^{1}$ Autism Spectrum Disorders Research Center, Hamadan University of Medical Sciences, Hamadan, Iran; ${ }^{2}$ Social Determinants of Health Research Center, Hamadan \\ University of Medical Sciences, Hamadan, Iran; ${ }^{3}$ Midwife in Social Security Organization, Hamadan, Iran
}

\begin{abstract}
Background: There is evidence of destructive effects of oxytocin use during labor on neurodevelopment.

Purpose: This meta-analysis pooled all observational studies to determine the association between labor induction and the risk of attention-deficit/hyperactivity disorder (ADHD) among children.

Methods: All observational studies (case-control and cohort) were reviewed until September 2020 after searches of the PubMed, Scopus, and Web of Science databases, the gray literature, and conference proceedings. The pooled odds ratios (ORs), relative ratios (RRs), and 95\% confidence intervals (CI (swere calculated as random effect estimates of association among studies.
\end{abstract}

Results: All observational studies were reviewed, including 4 cohort studies including 2,885,743 participants and 3 casecontrol studies including 51,135 participants (10,961 with ADHD and 40,174 in control groups) with a sample size of 2,936,878 participants. The pooled estimates of OR and RR did not indicate a significant association between labor induction and ADHD among children (OR, 1.13; 95\% CI, 0.90-1.35) and (RR, 1.10; 95\% CI, 0.96-1.24).

Conclusions: The findings showed that labor induction is not associated with an increased risk of ADHD among children. However, more studies are needed to investigate the relationship between labor induction and ADHD.

Keywords: Attention deficit disorder with hyperactivity, Labor induction, Meta-analysis

\section{Key message}

- This meta-analysis pooled all observational (case-control and cohort) studies to obtain the association between labor induction and the risk of attention-deficit/hyperactivity disorder (ADHD) among children.

- The findings showed that labor induction is not associated with increased risk of $\mathrm{ADHD}$ among children.

\section{Introduction}

Attention-deficit/hyperactivity disorder (ADHD) is a common neurodevelopmental disorder that occurs in approximately $3.4 \%$ of children worldwide. ${ }^{1)}$ One of the main features of ADHD is hyperactivity and impulsivity, which can lead to a lifelong disorder with significant social and economic burdens. ${ }^{2)}$

ADHD is an inherited disease because studies in twins have reported an inheritance estimate of about $76 \%{ }^{3)}$ Environmental factors including preterm delivery and poor fetal growth, exposure to cigarettes, prepregnancy body mass index, alcohol before delivery, and young parental age also increase the risk of ADHD. ${ }^{4,5)}$

There is evidence of destructive effects of oxytocin use during labor on neurodevelopment. Neonatal distress and hypoxia increase after labor induction due to hyperstimulation of the uterus $^{6}$ and can lead to possible physiological effects of oxytocin on brain development. ${ }^{7}$ It was hypothesized that the risk of neurodevelopmental disorders such as autism spectrum disorder and ADHD could increase with oxytocin use during labor. ${ }^{7}$

Some studies have reported an association between labor induction and $\mathrm{ADHD},{ }^{4,8)}$ while others have not. ${ }^{9,10)}$

To date, only Lønfeldt et al. ${ }^{11)}$ performed a meta-analysis of the association between labor induction and the risk of ADHD. The authors did not report a significant association between labor induction and the risk of ADHD (relative risk [RR], 1.17; 95\% confidence interval [CI], 0.77-1.78). However, this metaanalysis included only 3 studies. Therefore, we performed the present meta-analysis of all observational studies to determine the association between labor induction and the risk of ADHD among children.

\section{Methods}

\section{Data sources}

This meta-analysis was performed to determine the association between labor induction and the risk of ADHD among children.

Corresponding author: Saeid Bashirian, PhD. Social Determinants of Health Research Center, Hamadan University of Medical Sciences, Fahmideh Street, Hamadan, Iran 凶Email:S_bashirian@yahoo.com, Bashirian@umsha.ac.ir, https://orcid.org/0000-0003-2133-087X

Received: 13 September, 2020, Revised: 28 December, 2020, Accepted: 12 January, 2021

This is an open-access article distributed under the terms of the Creative Commons Attribution Non-Commercial License (http://creativecommons.org/licenses/by$\mathrm{nc} / 4.0 /$ ) which permits unrestricted non-commercial use, distribution, and reproduction in any medium, provided the original work is properly cited. Copyright (c) 2021 by The Korean Pediatric Society 
The systematic review used the published PRISMA (Preferred Reporting Items for Systematic Reviews and Meta-Analyses) statement checklist.

\section{Search strategy}

All published studies published through September 2020 were retrieved from the PubMed, Scopus, and Web of Science database, the gray literature, and conference proceedings. The index terms used in the present meta-analysis were: (induction or Pitocin or Syntocinon' or 'synthetic oxytocin) and (labor or labor) and (attention-deficit/hyperactivity disorder or ADHD or attention deficit disorder with hyperactivity or ADD or attention deficit disorder hyperactivity, attention deficit disorder hyperactivity, child attention deficit disorder). We then manually reviewed the reference lists of the retrieved articles and contacted the authors of the included articles to identify any other related articles.

\section{Inclusion and exclusion criteria}

In the systematic search, observational studies (case-control and cohort) that identified the association between labor induction and risk of ADHD among children were included. Labor induction in all studies was performed using oxytocin. Case reports, case series, reviews, and letters to the editor were excluded from the present meta-analysis.

\section{Data extraction}

The study titles, abstracts, and full texts were screened independently by 2 authors (EJ and BF). Any disagreement was resolved by the third author. The data sets extracted included publication year, first author, country, study design, ADHD diagnostic criteria, number of participants, OR, RR, and their associated $95 \% \mathrm{CI}$, adjustment, child age (range/mean), and quality.

\section{Quality assessment}

Study quality was assessed using the improved NewcastleOttawa Scale for assessing the risk of bias in observational studies. ${ }^{12)}$ Two investigators (EJ and BF) performed the quality assessment independently, and the scores of the studies were categorized as low quality ( $<7$ points) or high quality $(\geq 7$ points).

\section{Statistical analysis}

The pooled OR, RR, and 95\% CI were calculated from eligible studies used as random effect estimates of the association among studies.

Heterogeneity testing using $I_{2}$ statistics was performed to assess interstudy variance. We considered low, medium, and high heterogeneity as $I_{2}$ statistic values of $25 \%, 50 \%$, and $75 \%$, respectively.

To examine publication bias, Begg's and Egger's regression tests were performed. ${ }^{13)} P$ values of $<0.05$ were considered significant for publication bias. The present meta-analysis was performed using Stata 13 (StataCorp LP., College Station, TX, USA).

\section{Results}

\section{Study description}

A total of 252 studies were identified in the initial search; of them, 39 duplicate articles were excluded. The articles were reviewed by title, abstract, and then full text. Ultimately, 7 articles were included in the present analysis. The study selection process is presented in Fig. 1. The following studies were included: 4 cohort studies, $\left.{ }^{9}, 14-16\right)$ including 2,885,743 participants; and 3 case-control studies ${ }^{4,8,10)}$ including 51,135 participants $(10,961$ with ADHD and 40,174 in control groups) for a total sample size of 2,936,878 participants (Table 1). Silva et al. ${ }^{10)}$ reported the results separately for males and females.

The confounding variables of the association between labor induction and ADHD among children included maternal age, parity, 5-minute Apgar score, year of birth, mother's blood pressure, birth presentation, mode of delivery, smoking, psychiatric history, and gestational age.

\section{Main analysis}

We present the association between labor induction and ADHD among children in a forest plot in Fig. 2. The pooled estimates of $\mathrm{OR}$ and $\mathrm{RR}$ did not indicate a significant association between labor induction and ADHD among children (OR, 1.13; 95\% CI, 0.90-1.35 and RR, 1.10; 95\% CI, 0.96-1.24, respectively). There was medium heterogeneity among the studies reporting the risk of ADHD among children based on OR $\left(I_{2}=46.8 \%, P=0.111\right)$. There was high heterogeneity in studies reporting the risk of $\mathrm{ADHD}$ among children based on RR $\left(I_{2}=96.8 \%, P=0.000\right)$. There was no evidence of publication bias among the included studies. The $P$ values for Begg's and Egger's regression tests were 0.000 and 0.476 , respectively. Silva et al. ${ }^{10)}$ reported oxytocin induction in males (OR, 1.18; 95\% CI, 0.99-1.41) and females (OR, 1.07; 95\% CI, 0.74-1.55)

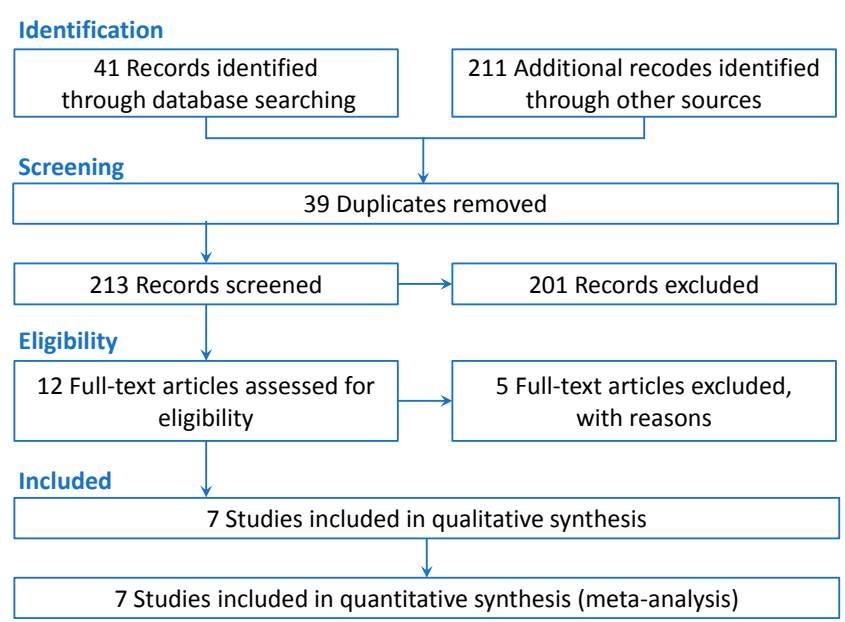

Fig. 1. Study selection process of the systematic review. 
Table 1. Summary of the included studies

\begin{tabular}{|c|c|c|c|c|c|c|c|c|}
\hline Study & Country & Design & Sample size & Estimate & Adjustment & $\begin{array}{c}\text { Age }(y r), \\
\text { range/mean }\end{array}$ & $\begin{array}{l}\text { Autism } \\
\text { criteria }\end{array}$ & Qualitya) \\
\hline Kurth $^{8)}(2012)$ & USA/Canada & Case-control & $\begin{array}{c}172 \\
\text { (88 cases \& } \\
84 \text { controls) }\end{array}$ & Odds ratio & Crude & $3-25$ & DSM-IV & Low \\
\hline Henriksen et al., ${ }^{9)}(2015)$ & Denmark & Cohort & 546,146 & Hazard ratio & Adjusted & No data & ICD-10 & High \\
\hline Wiggs et al., ${ }^{16)}(2017)$ & Sweden & Cohort & $1,574,204$ & Hazard ratio & Adjusted & $15-16$ & ICD & High \\
\hline Silva et al.. ${ }^{10)}(2014)$ & Australia & Case-control & $\begin{array}{c}1,430 \\
\text { (464 cases \& } \\
966 \text { controls) }\end{array}$ & Odds ratio & Adjusted & $1-17$ & DSM-IV, ICD-10 & High \\
\hline Curran et al.. ${ }^{14)}$ (2016) & Ireland & Cohort & 625,042 & Odds ratio & Adjusted & 7 & No criteria & High \\
\hline Stokholm et al., ${ }^{15)}(2020)$ & Finland/Denmark & Cohort & 140,351 & Hazard ratio & Adjusted & 2 and higher & ICD-9, 10 & High \\
\hline Sucksdorff et al.. ${ }^{4)}(2018)$ & Finland & Case-control & $\begin{array}{c}49,533 \\
(10,409 \text { cases } \& \\
39,124 \text { controls })\end{array}$ & Odds ratio & Adjusted & 2 and higher & ICD-9, 10 & High \\
\hline
\end{tabular}

DSM-IV, Diagnostic and Statistical Manual of Mental Disorders, fourth edition; ICD-10, International Classification of Diseases, tenth revision; ICD-9, International Classification of Diseases, ninth revision.

a) The study quality assessment was performed using the improved Newcastle-Ottawa Scale.

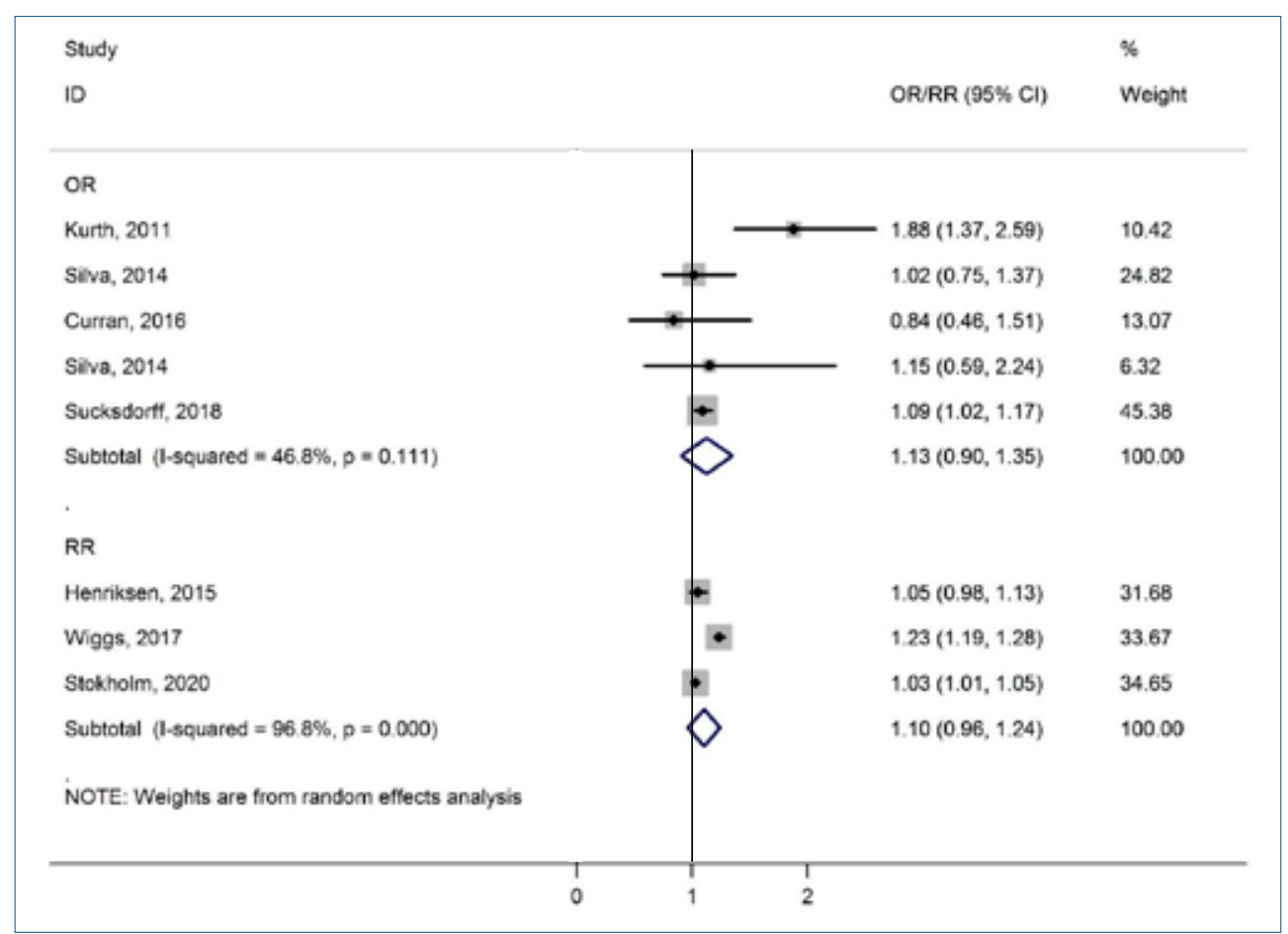

Fig. 2. Forest plot of the association between labor induction and attention-deficit/hyperactivity disorder. OR, odds ratio; $\mathrm{RR}$, relative risk; $\mathrm{Cl}$, confidence interval.

separately. Therefore, we display the data separately for males and females in Fig. 2.

\section{Sensitivity analysis}

There was high heterogeneity among studies assessing the association between labor induction and the risk of ADHD based on the pooled estimates of RR $\left(I_{2}=96.8 \%\right)$. Therefore, we conducted a sensitivity analysis based on the sequential algorithm to gain interstudy homogeneity. We obtained the minimum desired $I_{2}$ threshold (50\%) by omitting one study from the meta-analysis assessing the association between labor induction and the risk of ADHD. The removal of this reference ${ }^{16)}$ increased the heterogeneity to $6.4 \%$ (OR, 1.03; 95\% CI, 1.01-1.05; $P=0.000$ ).

\section{Study quality}

All studies in the present meta-analysis were of high quality except for one ${ }^{8)}$ according to the Newcastle-Ottawa Scale (Table $1)$.

\section{Discussion}

This meta-analysis showed no association between labor 
induction and the risk of ADHD among children. This metaanalysis included all 7 published case-control and cohort studies to assess the association between labor induction and the risk of ADHD among children. No significant association was reported between labor induction and the risk of $\mathrm{ADHD}$ among children. The pooled estimates of the included studies indicated that the $\mathrm{OR}$ and $\mathrm{RR}$ of $\mathrm{ADHD}$ among children after labor induction were 1.13 and 1.10 , respectively.

A meta-analysis study was performed by Lonfeldt et al. ${ }^{11)}$ in 2019. The authors reported a significant association between labor induction and the risk of ADHD (RR, 1.17; 95\% CI, 0.77-1.78). However, this meta-analysis included only 3 studies. These issues might introduce the possibility of selection bias.

There is evidence based on the important roles of endogenous oxytocin in the regulation of stress, anxiety, cognition, and social cognition and behaviors in humans. ${ }^{17)}$ In addition, animal studies have reported that neonatal exposure to synthetic oxytocin led to changes in social behavior and the oxytocinergic system in adults. ${ }^{18)}$

Overactive uterine activity, characteristic of oxytocin-induced hypertonic contractions, may create an unfavorable environment for fetal neural growth, especially in cases in which the contractions become too prolonged. ${ }^{8)}$

In fact, any association between oxytocin-induced labor and ADHD may be confounded by genetic or environmental vulnerability. ADHD is highly inherited, and a meta-analysis showed a consistent association between ADHD and single nucleotide polymorphisms in the oxytocin receptor gene. ${ }^{19)}$

The present meta-analysis has major strengths, including its comprehensive search and large pooled sample size in the metaanalysis. In addition, all studies in this meta-analysis except for one ${ }^{8)}$ were adjusted for some risk factors for labor induction.

However, we did not perform a subgroup analysis of factors such as dose-dependent effects of oxytocin due to the small number of included studies. This is the main limitation of the present meta-analysis. However, this meta-analysis did not show an association between labor induction and ADHD among children based on epidemiological studies with a sample size of 2,936,878 participants.

In conclusion, these findings showed that labor induction was not associated with an increased risk of ADHD among children. However, more studies are needed to investigate the relationship between labor induction and ADHD.

\section{Footnotes}

Conflicts of interest: No potential conflict of interest relevant to this article was reported.

Acknowledgments: This study was supported by Hamadan University of Medical Sciences ( 9907084773 and ethical committee code IR.UMSHA.REC.1399.526).

\section{References}

1. Polanczyk GV, Salum GA, Sugaya LS, Caye A, Rohde LA. Annual research review: a meta-analysis of the worldwide prevalence of mental disorders in children and adolescents. J Child Psychol Psychiatry 2015;56:345-65.

2. Doshi JA, Hodgkins P, Kahle J, Sikirica V, Cangelosi MJ, Setyawan J, et al. Economic impact of childhood and adult attention-deficit/hyperactivity disorder in the United States. J Am Acad Child Adolesc Psychiatry 2012; 51:990-1002.e2.

3. Sucksdorff M, Lehtonen L, Chudal R, Suominen A, Joelsson P, Gissler $\mathrm{M}$, et al. Preterm birth and poor fetal growth as risk factors of attentiondeficit/hyperactivity disorder. Pediatrics 2015;136:e599-608.

4. Sucksdorff M, Lehtonen L, Chudal R, Suominen A, Gissler M, Sourander A. Lower Apgar scores and Caesarean sections are related to attentiondeficit/hyperactivity disorder. Acta Paediatr 2018;107:1750-8.

5. Jenabi E, Bashirian S, Khazaei S, Basiri Z. The maternal prepregnancy body mass index and the risk of attention deficit hyperactivity disorder among children and adolescents: a systematic review and meta-analysis. Korean J Pediatr 2019;62:374.

6. Oscarsson ME, Amer-Wåhlin I, Rydhstroem H, Källén K. Outcome in obstetric care related to oxytocin use. A population-based study. Acta Obstet Gynecol Scand 2006;85:1094-8.

7. Wahl RUR. Could oxytocin administration during labor contribute to autism and related behavioral disorders?-A look at the literature. Med Hypotheses 2004;63:456-60.

8. Kurth L. Birthing the ADHD generation? Exploring the association between prenatal Pitocin ${ }^{\circledR}$ exposure and childhood ADHD onset. ADHD Rep 2012;20:7-9,15-6.

9. Henriksen L, Wu CS, Secher NJ, Obel C, Juhl M. Medical augmentation of labor and the risk of ADHD in offspring: a population-based study. Pediatrics 2015;135:e672-7.

10. Silva D, Colvin L, Hagemann E, Bower C. Environmental risk factors by gender associated with attention-deficit/hyperactivity disorder. Pediatrics 2014;133:e14-22.

11. Lønfeldt NN, Verhulst FC, Strandberg-Larsen K, Plessen KJ, Lebowitz ER. Assessing risk of neurodevelopmental disorders after birth with oxytocin: a systematic review and meta-analysis. Psychol Med 2019;49: 881-90.

12. Wells GA, Shea B, O'Connell D, Peterson J, Welch V, Losos M, et al. The Newcastle-Ottawa Scale (NOS) for assessing the quality of nonrandomised studies in meta-analyses [Internet]. Ottawa Hospital Research Institute; 2009 Sep 15 [cited 2014 Mar 25]. Available from: http://www. ohri.ca/programs/clinical_epidemiology/oxford.asp.

13. Egger M, Davey Smith G, Schneider M, Minder C. Bias in meta-analysis detected by a simple, graphical test. BMJ 1997;315:629-34.

14. Curran EA, Cryan JF, Kenny LC, Dinan TG, Kearney PM, Khashan AS. Obstetrical mode of delivery and childhood behavior and psychological development in a British cohort. J Autism Dev Disord 2016;46:603-14.

15. Stokholm L, Juhl M, Talge NM, Gissler M, Obel C, Strandberg-Larsen K. Obstetric oxytocin exposure and ADHD and ASD among Danish and Finnish children. Int JEpidemiol 2021;50:446-56.

16. Wiggs KK, Rickert ME, Hernandez-Diaz S, Bateman BT, Almqvist C, Larsson $\mathrm{H}$, et al. A family-based study of the association between labor induction and offspring attention-deficit hyperactivity disorder and low academic achievement. Behav Genet 2017;47:383-93.

17. Al-Ayadhi LY. Altered oxytocin and vasopressin levels in autistic children in Central Saudi Arabia. Neurosciences (Riyadh) 2005;10:47-50.

18. Lefevre A, Sirigu A. The two fold role of oxytocin in social developmental disorders: a cause and a remedy? Neurosci Biobehav Rev 2016;63:16876.

19. LoParo D, Waldman I. The oxytocin receptor gene (OXTR) is associated with autism spectrum disorder: a meta-analysis. Mol Psychiatry 2015; 20:640-6. 
How to cite this article: Jenabi E, Seyedi M, Bashirian S, Fereidooni B. Is there an association between labor induction and attention-deficit/hyperactivity disorder among children? Clin Exp Pediatr 2021;64:489-93. https://doi.org/10.3345/ cep.2020.01557 